\title{
CDCC del profesorado de Química sobre los conceptos cantidad de sustancia y mol
}

The teacher's of Chemistry DCK on the concepts quantity of substance and mol

$$
\text { García, Aldemar }{ }^{1} \text { y Parga, Diana }{ }^{2}
$$

${ }^{1}$ Departamento de Ciencias Naturales. Colegio El Jazmín. Candidato a magister en docencia de la química, UPN. ${ }^{2}$ Departamento de Química. Facultad de Ciencia y Tecnología. Universidad Pedagógica Nacional. aldemargarci@hotmail.com y dparga@pedagogica.edu.co

\section{Resumen}

Esta comunicación presenta los resultados finales de una investigación desarrollado durante los años 2007 y 2008 en la Universidad Pedagógica Nacional en Bogotá, para determinar las concepciones del profesorado de educación media de química, del Colegio el Jazmín, y las características del conocimiento didáctico del contenido curricular (CDCC) que tienen al diseñar currículos para enseñar los conceptos de cantidad de sustancia y mol. Se describe si sus concepciones están enmarcadas por el modelo teórico atomista o equivalentista, y se explica el origen de dichas concepciones. Se desarrolló una Trama Histórica / Epistemológica (THE) en relación con estos modelos teóricos y se diseñó una Unidad Didáctica (UD) para enseñar dichos conceptos. Este trabajo forma parte del proyecto del Centro de Investigación CIUP, de la UPN: DQU-025-07.

\section{Palabras claves}

Conocimiento didáctico del contenido curricular, diseño curricular, teoría atomista, teoría equivalentista, mol, cantidad de sustancia, tramas histórico epistemológicas.

\section{Objetivos}

1. Caracterizar el conocimiento didáctico del contenido curricular (C.D.C.C.) del profesorado de química en ejercicio, cuando hacen diseños curriculares para enseñar los conceptos cantidad de sustancia y Mol.

2. Describir si las concepciones del profesorado de química están dentro del modelo atomista 0 equivalentista, y explicar el origen de dichas concepciones.

3. Desarrollar una Trama Histórica - Epistemológica (THE) en relación con los modelos teóricos del atomismo y equivalentismo.

4. Diseñar a partir de la THE, una Unidad Didáctica para enseñar los conceptos de cantidad de sustancia y su unidad el Mol.

\section{El Conocimiento Didáctico del Contenido Curricular CDCC}

De acuerdo con Garritz y Trinidad - Velasco (2004) en el verano de 1983, Lee S. Shulman dictó una conferencia en la Universidad de Texas, en Austin, la cual tituló ambiguamente "El paradigma perdido en la investigación sobre la enseñanza". Esta propuesta sorprendió a todo mundo ya que hasta entonces los estudios sobre la enseñanza se habían enfocado en las formas de comportamiento del profesor más que en las de su pensamiento. En 1986 Shulman pública Those who understand; Knowledge Growth in teaching y Knowledge and Teaching: foundations of the 
new reform en 1987. Shulman propone y desarrolla el concepto del conocimiento pedagógico del contenido (CPC) junto con sus colegas en el marco del Knowledge Growth in Teaching Project Este proyecto estudiaba como los maestros noveles adquirían nuevos conocimientos sobre el contenido y como esos nuevos conocimientos influían en la manera como ellos enseñaban.

El conocimiento didáctico del contenido curricular (CDCC) nace como una de las tres ramas del conocimiento pedagógico del contenido; Mora (1999), cita que el CDCC en la línea de investigación en la didáctica de las ciencias, ha propendido por hacer diferencias entre el saber especifico de las ciencias y el saber pedagógico/didáctico asociado con su enseñanza, convirtiéndose este último en un dominio especifico del conocimiento profesional del profesorado. Entre los objetivos de investigación en la línea del CDCC, Parga y Martinez (2007) destacan los siguientes:

Determinar las características que deben tener los contenidos de enseñanza y cómo se deben organizar en tomo al conocimiento escolar que permitan favorecer una evolución significativa de las concepciones del estudiantado (Mora y Parga, 2008). También el cómo diseñar los currículos de los espacios académicos o "asignaturas" en tomo a tramas conceptuales evolutivas cada vez más complejas, para la cual es fundamental el soporte de la historia y epistemología de las ciencias y en particular de la química, que permitan, desde el contexto de la didáctica, entender el curníalo como hipótesis progresivas de intervención - innovación e investigación escolar.

La contextualización didáctica de los estudios sobre el diseño curricular de los contenidos para la enseñanza de la química posee un espacio de intersección interdisciplinar entre cuatro grandes áreas (o esferas del conocimiento): primero, el conocimiento disciplinar (que podríamos llamar conocimiento químico de la química); segundo, el conocimiento histórico - epistemológico y social (conocimiento metadisciplinar de la química); tercero, la psicología del aprendizaje 0 psicopedagógico; y cuarto, el conocimiento del contexto escolar donde se realiza la labor docente.

\section{Dificultades en la enseñanza y desarrollo histórico en torno a los conceptos cantidad de sustancia y mol}

En la actualidad, la enseñanza de la única magnitud propia de la química que es la cantidad de sustancia y su unidad el mol, presenta varios problemas como lo afirman Mora y Parga (2005), de los cuales se destacan los siguientes:

$>$ Entendimiento inadecuado e insuficiente del concepto por parte del profesorado, y requisitos previos (químicos / matemáticos) no adquiridos.

$>$ Inconsistencias entre el docente, el libro de texto y la definición científica.

> Ninguna distinción ni relación entre los conceptos cantidad de sustancia y mol.

> Ninguna perspectiva histórica, discusión de su utilidad, ni acercamiento cualitativo.

> Muchas nociones frecuentemente desarrolladas que deben relacionarse (volumen, masa, número de moles, número de partículas, molaridad, número de Avogadro, volumen molar).

Azcona (1997), establece que entre el profesorado que enseña la química en básica secundaria y media, desconoce el desarrollo histórico de los conceptos cantidad de sustancia y mol, precisando que éstos surgen desde la confrontación entre los modelos teóricos atomista (Dalton) equivalentista (Richter) en el siglo XIX. La introducción del concepto Mol a la química ocurre en 1865 por el químico August Hofmann. En 1900 Wilhelm Ostuald define este concepto desde el modelo equivalentista, y en 1908 se afianza el modelo atomista ante los descubrimientos de las partículas subatómicas que conforman el átomo y el comportamiento molecular (Moreno, 2006). Furió, C., Padilla, K, (2003) citan que el mol fue finalmente definido como lo conocemos hoy por la IUPAC en 1961 como concepto atomista, y definido como unidad de la magnitud cantidad de sustancia en 1967. 


\section{La Trama Conceptual (TC), y la Unidad Didáctica (UD)}

Las tramas conceptuales se describen de acuerdo con Mora, García y Mosquera (2002) como aportaciones del análisis didáctico de las disciplinas científicas que resultan útiles para la determinación del conocimiento escolar a enseñar. Son un instrumento para organizar los conocimientos que se van a trabajar para luego ser articulados a unidades didácticas (como forma de organización y operacionalización del currículo); a partir de las tramas conceptuales se ponen en manifiesto las interacciones entre los contenidos, cosa que no lo logran las listas de las dásicas programaciones.

Según Mora y Parga, (2005) basados en las investigaciones de Martín del Pozo (1994) y GaráaDíaz (2004), el diseño curricular se puede operacionalizar en tomo a la trama conceptual evolutiva, para el diseño de secuencias de estrategias didácticas. Éste está articulado en:

1. Tramas histórico - epistemológicos que nos permiten ver la evolución de los conceptos y teorías en disputa, especificando el tipo de cambio ocurridos en la ciencia y ofreciendo descripciones de momentos histónicos que son particularmente ilustrativos de concepciones y obstáculos epistemológicos que inciden en la enseñanza escolar.

2. Tramas didácticos, fundamentados en los tramas histórico - epistemológicos que permiten establecer contenidos aptos para la enseñanza a nivel conceptual, procedimental y actitudinal.

La unidad didáctica es un instrumento de enseñanza, son unidades de programación, de diseño curricular y desarrollo de la enseñanza, y por tanto son una forma de organizar los programas escolares dotados de capacidad de integrar contenidos diversos y de estructural periodos relativamente largos de la actividad escolar. El diseño de unidades didácticas se asume como proyecto curricular en profundidad, planteado como hipótesis, que orienta y facilita el desarrollo practico, y obedecen a una necesidad sentida de un colectivo de profesionales de la educación y la enseñanza de las disciplinas al interior de una institución escolar (Mora, García y Mosquera, 2002), .

\section{Metodología}

El trabajo de esta investigación se rige desde los parámetros de la metodología cualítativa, y sigue el diseño de estudio de casos. La muestra de esta investigación es de tipo homogéneo, y se contó con siete profesores del área de ciencias naturales de educación básica secundaria y media del colegio Distrital El Jazmín en Bogotá (Colombia), que nos permitieron conocer, comparar, y evaluar las similitudes y diferencias entre su CDCC desde su manejo disciplinar, histórico epistemológico, y psicopedagógico de los conceptos cantidad de sustancia y Mol. El tiempo usado en esta investigación fue 2 años, desde Marzo de 2007 hasta Abril de 2009.

Los siguientes son las etapas que se siguieron para llevar a cabo esta investigación:

1. Diseño y aplicación al profesorado de una encuesta sobre los libros de texto de química usados para su diseño curricular para la enseñanza de los conceptos cantidad de sustancia y mol.

2. Diseño y aplicación de tres encuestas trabajadas sobre los libros de texto de química desde los aspectos disciplinares, históricos, y didácticos.

3. Diseño y aplicación al profesorado de cinco encuestas, la primera sobre CDCC desde la enseñabilidad de los contenidos y el diseño curriaular en general. Tres aplicadas sobre los conceptos cantidad de sustancia y mol, desde los aspectos disciplinares, históricos, y didácticos. La última aplicada para conocer impresiones sobre la THE y aportes para el diseño de la UD. 
4. Se hicieron dos entrevistas al profesorado para identificar aspectos de los modelos atomista y equivalentista, y las dificultades que se presentan en los procesos de enseñanza y aprendizaje de los conceptos cantidad de sustancia y mol.

5. Construcción de dos tramas conceptuales, cada una de un modelo teórico: atomismo y equivalentismo, siguiendo el orden de la dinámica cient́fica de Anna Estany (1990) combinados con los planteamientos de Root-Berstein (1984) y Richard Duschl (1987).

6. Construcción de la trama conceptual de los conceptos cantidad de sustancia y mol, de acuerdo con los conceptos aplicados en la química a nivel macroscópico y microscópico que éstos conforman.

7. Elaboración de la unidad didáctica de acuerdo con los siguientes parámetros: primero a los objetivos propuestos por Furió, Azcona y Guisasola (2006) para facilitar la comprensión de los conceptos cantidad de sustancia y mol, segundo los aportes obtenidos en la última encuesta con el profesorado, y tercero, diseñado sobre el modelo EpC (enseñanza para la comprensión).

\section{Conclusiones}

A partir de los datos obtenidos en los instrumentos aplicados al profesorado podemos conduir:

1. A partir del análisis de las pruebas iníciales sobre CDCC, el diseño curricular del profesorado de química está enfocado al conocimiento disajplinar y en menor grado al conocimiento didáctico, dejando de lado los otros componentes del CDCC, como los son el histórico - epistemológico, psicopedagógico y del contexto escolar.

2. Se manifiesta el desconocimiento de la magnitud cantidad de sustancia quizá por el poco trabajo de este conceptos en la formación docente, poca citación (simplista) en los libros de texto usados para su diseño curricular, y a que en los sus aursos de postgrado no están enfocados a la química y su enseñanza.

3. La terminología usa por el profesorado para definir el concepto mol nos muestra el predominio del modelo atomista, pero dicha terminología es citada desde un punto de vista macroscópico, por eso el diseño curricular enfatiza en lo perceptible.

4. Con gran preocupación sigue apareciendo tanto en el profesorado como los libros de texto usados para su diseño curricular, la citación y aplicación de conceptos en desuso en la química, como algunos términos equivalentistas que prevalecen en la enseñanza como la normalidad y el equivalente gramo, y otros como el átomo gramo, molécula gramo, y peso molecular, los aules fueron derogados y actualizados por la IUPAC hace más de 20 años.

5. Los resultados obtenidos de los diversos instrumentos aplicados nos permiten definir que el diseño curricular hecho por el profesorado para la enseñanza del concepto mol es enfocado desde la aplicación de ejemplos y ejercicios algońtmicos, mostrándonos que son pocas las estrategias didácticas aplicadas y que esto afecta el proceso de aprendizaje del estudiantado, y el desconocimiento del concepto cantidad de sustancia, su uso y relación con el concepto mol.

6. El conocimiento de la trama histónica - epistemológica (THE) por parte del profesorado, permitió que ellos conocieran el conflicto de los modelos atomista - equivalentista (que en su mayoría desconocían), corregir errores históricos en la enseñanza de la química (en especial del concepto mol, número de Avogadro y volumen molar). El profesorado conduyó que los avances de la química no son lineales, ni espontáneos, muestra que los procesos que permiten ratificar una teoría son bastante extensos, rigurosos y llenos de contradictores, e interesarse más en momentos históricos del desarrollo de la química como ciencia, para ser discutidos en el aula como 
introducción a iniciar cada tema, en vez de la simple enseñanza bibliográfica de algunos químicos que es lo que tradicionalmente se ha enseñado.

7. La trama conceptual (TC) permitió que el profesorado presentara una transformación de los significados sobre el concepto mol (más apropiados para el modelo atomista), desde el conocimiento de las diversas aplicaciones que de éste poseen tanto a nivel macroscópico, como a nivel microscópico, que sus aplicaciones no solo son explíatas para la química sino para las otras ciencias naturales, dando al concepto una aplicación transversal.

8. La elaboración de la unidad didáctica (UD) de los conceptos cantidad de sustancia y mol, muestra al profesorado nuevas estrategias didácticas en las enseñanza de estos conceptos, además la aplicación de los conceptos en los procesos de enseñanza - aprendizaje son desarrollados a largo plazo.

\section{Bibliografía}

Furió C., Azcona, R. y Guisasola, J. (2006). Enseñanza de los conceptos de cantidad de sustancia y de MOL basada en un modelo de aprendizaje como investigación orientada. Enseñanza de las Ciencias. 24 (1), $43-58$.

Furió, C., Padilla, K. (2003). La evolución histórica de los conceptos científicos como prerrequisito para comprender su significado actual: el caso de la "cantidad de sustancia" y el "mol". En Didáctica de las ciencias experimentales y sociales, No. 17, 55 - 74.

Garća-Díaz, E. (2004). Educación ambiental, constructivismo y complejidad. Sevilla, Díada Editora.

Garritz y Trinidad - Velasco (2004). El conocimiento pedagógico del contenido. En Educación Química, Vol. 15, No. 2, 2004, 98-102

Martín del Pozo, R, (1994). El Conocimiento del Cambio Químico en la Formación Inicial del Profesorado. Estudio de los Conceptos disciplinares y didádicas de los Estudiantes del Magisterio. Tesis Doctoral Inédita. Universidad de Sevilla España.

Mora, W. (1999). Elementos para la Renovación Curricular de los Proyectos de Formación del Profesorado de Química: Una Propuesta desde la Pedagogía y la Didáctica como Disciplinas Fundantes. En Educación y Pedagogía. Universidad de Antioquia: Vol. XI, No. 25, 121 - 145.

Mora, W., Garáa. A., Mosquera, C. (2002). Bases para la construcción de un auerpo conceptual didáctico del desarrollo histónico - epistemológico de los conceptos estructurantes de la química. Revista Científica. 259 - 286. Universidad Distrital Francisco José Caldas.

Mora, W., Parga, D. (2005). De las investigaciones en preconcepciones sobre mol y cantidad de sustancia, hacia el diseño curricular en química. En Educación y Pedagogía, Sep-Dic. Vol. XVII. No. 43, 164-175

Mora, W., Parga, D. (2007). Las tramas histórico - epistemológicas en el contexto del CDCC: el diseño de niveles de formulación de la construcción del modelo teórico estructural en química orgánica. En Tecné, Episteme y Didaxis: TED, Número 21, p. 100 -108.

Moreno A. (2006). Atomismo versus Energetismo: controversia científica a finales del XIX. En Enseñanza de las Ciencias, 24 (3), 411 - 428.

Mora, W y Parga, D. (2008). El Conocimiento Didáctico del Contenido en Química: De las Tramas Histórico/Epistemológicas a las Tramas de Contexto / Aprendizaje. Tecnè, Episteme $y$ Didaxis. TED. No. 24. Pp. 54-74.

Parga, D. y Martínez, L. (2007). Conocimiento didáctico del contenido curricular en química: una estratega sustentada en el diseño de tramas conceptuales. Universidad Pedagógica Nacional. Colombia. Proyecto de investigación CIUP-DQU-025-07. 